\title{
Effect of mitratapide on body composition, body measurements and glucose tolerance in obese Beagles
}

\author{
Britta Dobenecker • Manuelle De Bock • Marc Engelen • \\ Lieve Goossens • Armin Scholz • Ellen Kienzle
}

Accepted: 1 June 2009 / Published online: 20 June 2009

(C) The Author(s). 2009 This article is published with open access at Springerlink.com

\begin{abstract}
The objective of this study was to confirm that weight loss after treatment with mitratapide (Yarvitan ${ }^{\circledR}$ ) is loss of adipose tissue. Obese dogs were treated with the recommended treatment schedule of mitratapide. Dual-energy X-ray absorptiometry (DEXA) was done before and after the treatment schedule. Body weight, feed consumption and pelvic circumference were recorded and a glucose tolerance test was performed. Dualenergy X-ray absorptiometry measurements showed an impressive loss of fat tissue, corresponding to a mean loss of approximately $41.6 \%$ of the body fat mass recorded before treatment. After treatment with mitratapide, the mean body fat percentage had returned within the normal range. At the end of the study, the dogs had lost on average $14.2 \%$ of their body weight and $15.2 \%$ of their pelvic circumference compared to baseline. The results also suggest that losing weight with mitratapide might help to reverse insulin resistance.
\end{abstract}

Keywords Mitratapide $\cdot$ Dog $\cdot$ Body fat $\cdot$ Dual-energy X-ray absorptiometry

\section{Introduction}

Canine obesity is the most common form of malnutrition encountered in small animal practice in the western world (Burkholder and Toll 1997). Obesity is caused by an energy intake in

B. Dobenecker $(\bowtie) \cdot$ E. Kienzle

Department of Veterinary Sciences, Chair of Animal Nutrition and Dietetics, Faculty of Veterinary Medicine, Ludwig-Maximilians University Munich, Schönleutnerstrasse 8, 85764 Oberschleissheim, Germany

e-mail: Dobenecker@1rz.uni-muenchen.de

M. De Bock $\cdot$ M. Engelen $\cdot$ L. Goossens

Janssen Animal Health, Division of Janssen Pharmaceutica NV, Turnhoutseweg 30, 2340 Beerse, Belgium

A. Scholz

Livestock Center Oberschleissheim, Faculty of Veterinary Medicine, Ludwig-Maximilians University Munich, Sankt-Hubertus-Strasse 12, 85764 Oberschleissheim, Germany 
excess of the requirement, resulting in a positive energy balance and accumulation of adipose tissue (body fat). Incidence rates are estimated around 20-41\% (Edney and Smith 1986; Burkholder and Bauer 1998; McGreevy et al. 2005) and are following the same rising trend as human obesity. Overweight and obesity have detrimental effects on general health (Burkholder and Toll 1997). Typical consequences and co-morbidities of overweight and obesity are diabetes mellitus, osteoarthritis, respiratory problems, cardiovascular disease, neoplasia, hepatic disease, anesthetic complications and dermatology problems (Burkholder and Toll 1997). Specific studies have also indicated that overweight and obesity have a direct impact on longevity (Kealy et al. 2002; Lawler et al. 2005).

In human medicine, numerous studies illustrate the health benefit of weight loss (Wing et al. 1987; Goldstein 1992; Mertens and Van Gaal 2000; Pi-Sunyer 1996). Even modest weight loss has been demonstrated to provide a clear health benefit. The number of studies on this subject in dogs is rather limited, but the general principles can be extrapolated from human data.

Precise measurements of body composition are an added value in studies of obesity and other nutritional disorders. The three-compartment model using dual-energy X-ray absorptiometry (DEXA) shows a good and close correlation with dissection and fat analysis. DEXA can estimate three body compartments consisting of fat mass, lean body mass and bone (Speakman et al. 2001).

Treatment of canine overweight and obesity traditionally consists of dietary management in combination with exercise (Burkholder and Toll 1997, Burkholder and Bauer 1998). However, this requires long-term motivation of the owner and because there is no short-term visible effect, it might be rather frustrating. Recently a new product (Yarvitan ${ }^{\circledR} 5 \mathrm{mg} / \mathrm{ml}$, Janssen Pharmaceutica NV, Beerse, Belgium) containing mitratapide as active ingredient was approved in the EU for the management of overweight and obesity in dogs. Mitratapide belongs to the class of microsomal triglyceride transfer protein (MTP) inhibitors. Orally administered mitratapide inhibits microsomal triglyceride transfer protein at the level of the enterocytes resulting in a decreased uptake of dietary lipids.

The objective of this study was to compare the body composition of obese Beagle dogs before and after treatment with mitratapide by means of DEXA. Body weight, feed consumption, pelvic circumference and glucose tolerance were also evaluated during the experiment.

\section{Material and methods}

Investigational animals

Six intact female Beagles were included in this experiment. The animals were between two and six years of age. Five months prior to the start of the study, the dogs were put on a high fat diet and were fed ad libitum during three hours a day. At the start of the treatment, they were obese, but otherwise healthy with a body weight of at least $20 \%$ above their ideal body weight. The dogs were housed in cages in groups of two animals. During feeding, they were housed individually. During approximately four hours per day, the dogs were kept outdoors in kennels in groups of two to four animals. The level of exercise during the study was not higher than before the study. The dogs had free access to drinking water throughout the entire experiment.

The study was conducted according to all German regulations concerning ethics and with the utmost respect for animal welfare under constant veterinary supervision. 
Study design and treatment

This study was a longitudinal study with six dogs and comprised of a mitratapide treatment schedule ( 8 weeks). The study was designed in such a way that each dog served as its own control on the basis of baseline values (body weight, DEXA, feed consumption and pelvic circumference) and historical values (insulin/glucose). The mitratapide treatment schedule was as follows: the dogs were treated with mitratapide oral solution (Yarvitan ${ }^{\circledR} 5 \mathrm{mg} / \mathrm{ml}$; Janssen Pharmaceutica NV, Beerse, Belgium) at the therapeutic daily dose of $0.63 \mathrm{mg} / \mathrm{kg} /$ day. All dogs were treated once daily for two times 21 consecutive days ( 3 weeks) with an intermediate period of 14 consecutive days ( 2 weeks) without treatment.

During the first three weeks of the mitratapide treatment schedule, the dogs were fed ad libitum a standard dry feed (Frolic ${ }^{\mathrm{TM}}$, Mars, Germany, metabolizable energy (ME) $3240 \mathrm{kcal} / \mathrm{kg}$ ) during three hours a day. Afterwards the dogs received an amount of feed in accordance with their maintenance energy requirements (MER) during three hours a day until the end of the study. Being seriously overweight the dogs were considered inactive dogs. MER was calculated using the recommendations for inactive dogs $(95 \mathrm{kcal} / \mathrm{kg}$ body weight $^{0.75}$, NRC 2006) based on the ideal body weight.

\section{Evaluation criteria}

DEXA was performed under anaesthesia to assess the dog's body composition on Day -1 and Day 56. The whole body was examined ventrodorsally (Fig. 1).

The body weight of each dog was recorded on Day -2, 1, 21, 35, 36 and 56. Pelvic circumference was measured on Day $-1,21,35$ and 56. This was done with a measuring tape with an accuracy of $0.1 \mathrm{~cm}$. Pelvic circumference is a morphometric measure that has been demonstrated to be well correlated with the percentage of body fat in dogs (Burkholder and Toll 1997).

A glucose tolerance test and measurement of insulin levels was performed on Day -2 and Day 55. The dogs were injected intravenously with a $40 \%$ glucose solution $(1 \mathrm{~g} / \mathrm{kg}$ body weight, Fa. Delta Select GmbH, Dreieich, Germany). Before injection and 15, 30, 45 and 60 minutes after injection each time two blood samples were taken. One blood sample was used to analyse glucose (Hitachi 911 Chemistry Analyzer, Roche). Serum from the other

Fig. 1 Experimental setup for DEXA measurements in the $\operatorname{dog}$

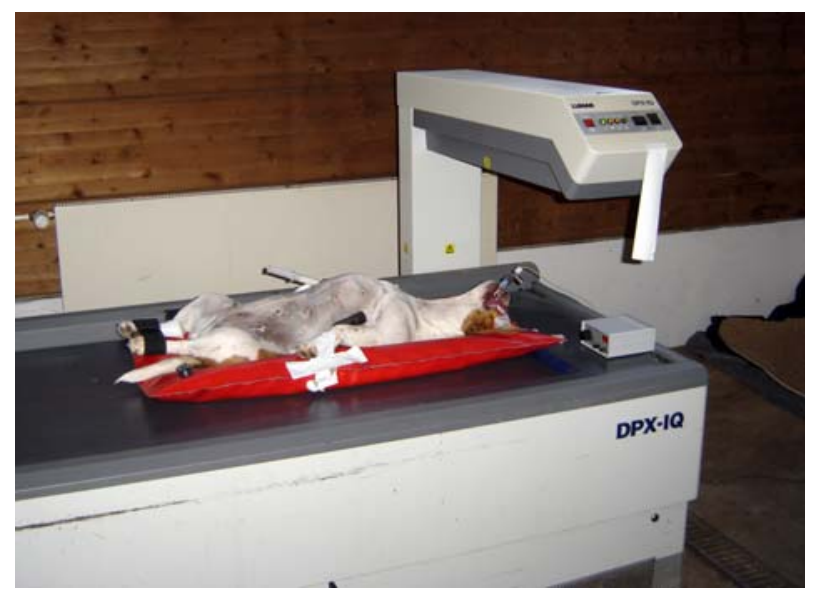


Table 1 DEXA measurements and statistical comparison

\begin{tabular}{lllll}
\hline Period & & Fat mass [g] & Lean body mass [g] & $\begin{array}{l}\text { Bone mineral } \\
\text { content }[\mathrm{g}]\end{array}$ \\
\hline Baseline (Day -1) & Mean \pm SD & $5824 \pm 1391$ & $12272 \pm 940$ & $605 \pm 43$ \\
& Median (Min-Max) & $5965(4148-7447)$ & $12018(11266-13912)$ & $597(522-674)$ \\
End study (Day 56) & Mean \pm SD & $3398 \pm 1339$ & $11923 \pm 665$ & $582 \pm 32$ \\
& Median (Min-Max) & $2718(2173-5507)$ & $11685(11348-13068)$ & $569(553-635)$ \\
P-value (Day -1 vs Day 56) & 0.0313 & 0.3125 & 0.0625 \\
\hline
\end{tabular}

blood sample was used for an insulin radio immuno assay. The insulin sensitivity index was calculated according to Belfiore et al. (2001).

Daily feed uptake of each dog was recorded and all dogs were observed from Day -2 until Day 56 for abnormal behaviour and adverse events.

\section{Statistical analysis}

The experimental unit was each individual dog. The statistical tests were two-sided with a significance level of $5 \%$. Non-parameteric tests were most appropriate for parameters expressed as scores and for parameters expressed on a continuous scale, in view of the number of dogs in the study. Intra-statistical comparisons were applied on the individual values by comparisons between the baseline (Day -2 or Day -1) and Day 56 of the experiment by means of Wilcoxon Signed Rank test.

Fig. 2 DEXA outprint of the body composition of a dog before the start of the mitratapide treatment schedule (a) and at the end of the mitratapide treatment schedule (b)

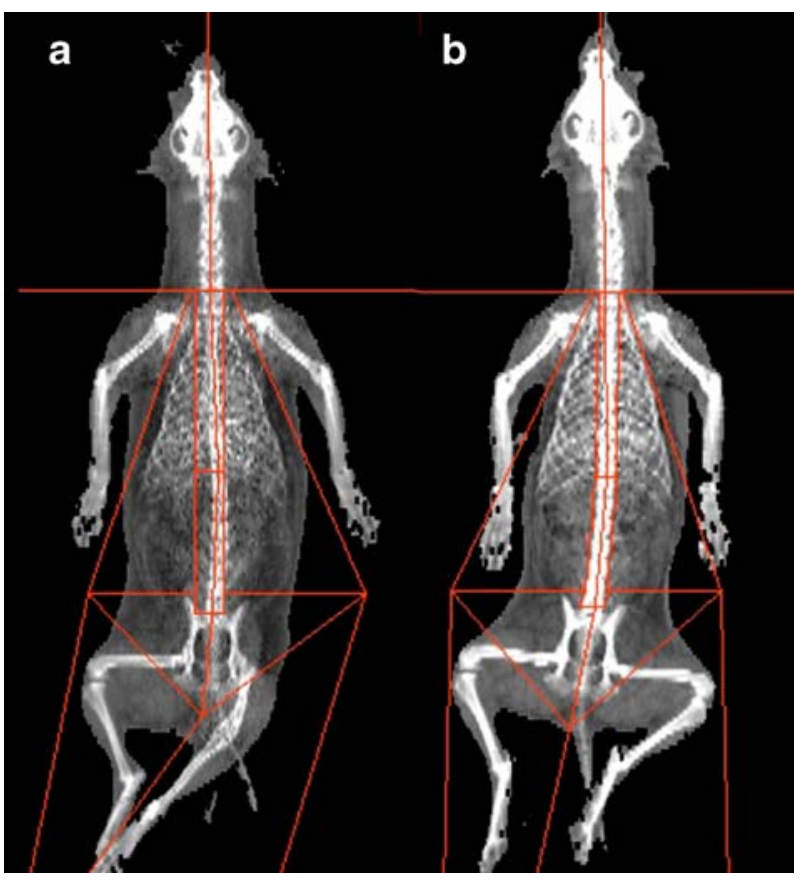




\section{Baseline (Day -1)}

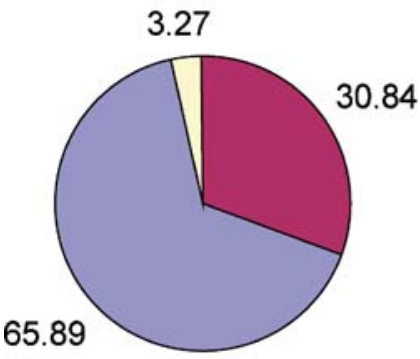

\section{End of treatment (Day 56)}

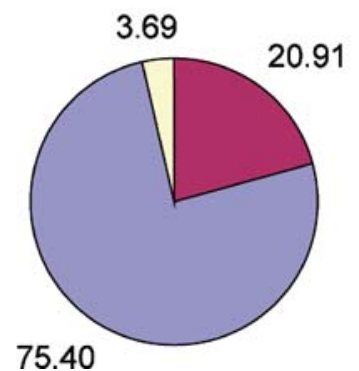

Fat mass

Lean body mass

Bone

Fig. 3 Mean (\%) DEXA measurements (fat mass, lean body mass, bone) versus total before and after the treatment schedule

\section{Results}

DEXA measurements (Table 1, Fig. 2) show impressively how the fat mass declined whereas the bone and lean body mass were hardly affected. The dogs lost a clinically relevant amount $(\mathrm{g})$ of fat mass during the trial, corresponding to a loss of approximately $41.6 \%$ of their initial body fat mass. The percentage of body fat (Fig. 3) after the mitratapide treatment schedule is significantly lower compared to Day $-1 \quad(p=0.0313)$.

Table 2 clearly shows that the mean body weight decreased over time compared to baseline. The mean weight loss of the dogs at the end treatment compared to baseline was $14.2 \%$ (range: $8.0 \%-23.1 \%$; Fig. 4) and this was statistically significant $(p=0.0313)$. In Table 2 it is also shown that the mean pelvic circumference decreased over time compared to baseline. The decrease of pelvic circumference (15.2\%, Fig. 4) at the end of treatment was also statistically significant $(\mathrm{p}=0.0313)$ compared to baseline.

The glucose tolerance test showed that five out of six dogs had decreased insulin sensitivity at baseline. After the mitratapide treatment schedule, in four of these five dogs, an increase of insulin sensitivity was noticed (Table 3, Fig. 5).

Records were made of the individual feed uptake of each animal. It was noticed that during the study, the dogs ate less during periods with mitratapide treatment than during the two-week treatment-free interval (Fig. 6).

Table 2 Body weight, body fat and pelvic circumference data

\begin{tabular}{llll}
\hline Parameter & & Baseline (Day -2) & End study (Day 56) \\
\hline Body weight (kg) & Mean \pm SD & $18.3 \pm 2.13$ & $15.7 \pm 1.71$ \\
Weight loss (\%) & Median (Min-Max) & $17.8(16.3-21.8)$ & $15.0(14.4-18.9)$ \\
& Mean \pm SD & - & $14.2 \pm 4.89$ \\
Mean body fat (\%) vs. total & Median (Min-Max) & - & $13.5(8.0-23.1)$ \\
& Mean \pm SD & $30.8 \pm 4.78$ & $20.9 \pm 5.98$ \\
Pelvic circumference (cm) & Median (Min-Max) & $32.2(25.0-36.1)$ & $18.3(14.4-28.8)$ \\
& Mean \pm SD & $58.7 \pm 4.37$ & $49.9 \pm 7.81$ \\
Pelvic circumference loss (\%) & Median (Min-Max) & $58.5(54.0-64.0)$ & $46.0(43.5-63.0)$ \\
& Mean \pm SD & - & $15.2 \pm 8.29$ \\
& Median (Min-Max) & - & $16.7(1.6-25.0)$ \\
\hline
\end{tabular}


Fig. 4 Mean body weight loss $(\%) \pm \mathrm{SD}$ and mean pelvic circumference loss $(\%) \pm \mathrm{SD}$ over time

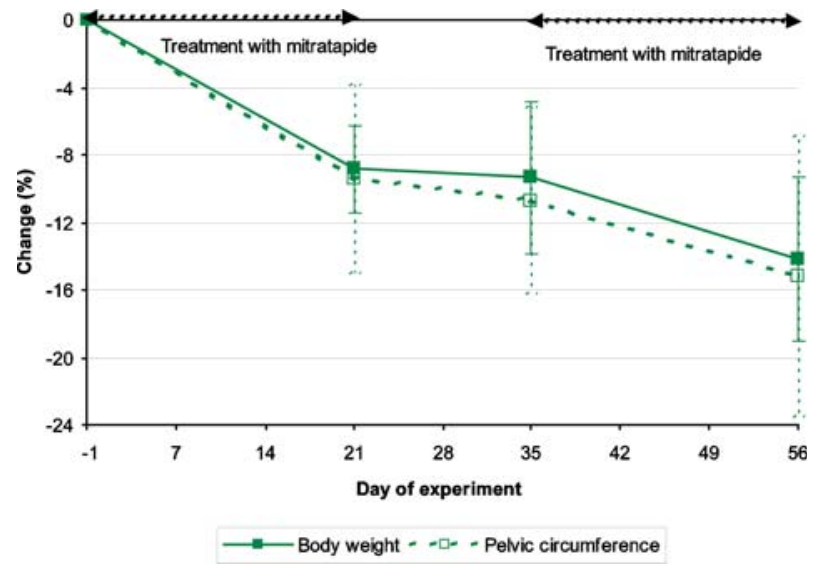

Reduced faeces quality and vomiting of ingested non-feed materials was seen from time to time during treatment. Incidental vomiting was recognized once; no tracking to an individual was possible, though. Such observations were also made during periods without treatment. There was one case of otitis externa and one case of pseudopregnancy.

\section{Discussion}

The functions of body fat have traditionally been understood as energy storage, thermal insulation and structural support for some organs. However, it has recently been shown that adipose tissue is very metabolically active, produces several hormones and constitutes the largest endocrine organ in the body (Armstrong and Churchill 2008; Greco 2008). Both the amount and distribution of adipose tissue plays a role in insulin resistance and other obesity related disorders. Returning dogs to optimal body condition and normalizing the percentage body fat, i.e. between 12.3 and 27.5\% (Laflamme 1997), will therefore significantly improve control of many obesity related diseases.

The primary objective of this study was to demonstrate that weight loss after treatment with mitratapide is loss of adipose tissue. The measurements of body composition using DEXA showed an impressive, clinically relevant reduction of body fat in all dogs (41.6\%). After the mitratapide treatment schedule, the mean body fat percentage had returned within the normal range (i.e. between 12.3 and $27.5 \%$ body fat). This was the case in four out of six dogs. The two other dogs had a fat percentage of $28 \%$ and $28.8 \%$, respectively, which is only slightly higher than the normal range. These two dogs, however, had a much higher fat percentage than the other dogs at the start of the study. Lean body mass and bone mineral content remained more or less constant. These DEXA results show that mitratapide does not induce weight loss by reduction of lean tissue but by reduction of fat tissue. These

Table 3 Insulin sensitivity index

\begin{tabular}{llll}
\hline & Historical data (ideal BW) & Start study Day -2 (obese) & End of treatment Day 56 \\
\hline Mean \pm SD & $1.04 \pm 0.169$ & $0.75 \pm 0.271$ & $0.91 \pm 0.201$ \\
Median (Min-Max) & $1.02(0.8-1.3)$ & $0.71(0.4-1.1)$ & $0.92(0.6-1.2)$ \\
\hline
\end{tabular}


Fig. 5 Insulin sensitivity before and after treatment with mitratapide (Value: 1 = normal, $<1=$ decreased insulin sensitivity (Belfiore et al. 2001))

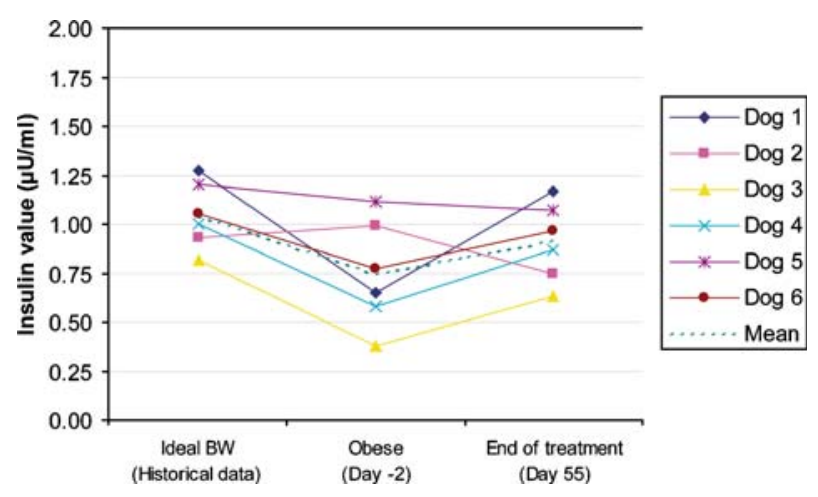

findings were statistically significant, even based on a small sample of six dogs, with all dogs responding to treatment as reflected by all evaluated parameters.

The body measurement parameters, i.e. body weight and pelvic circumference at the end of the study, demonstrate a clinically relevant and statistically significant loss of body weight $(14.2 \%$, range $8.0 \%-23.1 \%)$ and pelvic circumference $(15.2 \%$, range $1.6 \%-25.0 \%)$ compared to baseline.

The lower feed consumption observed during the mitratapide treatment was expected and is related to the mode of action of MTP inhibitors. Mitratapide blocks the microsomal triglyceride transfer protein that is normally involved in the absorption of dietary fats. By inhibiting this protein, mitratapide reduces the amount of fat absorbed through the intestines. Fat sequestered in the enterocyte is also believed to cause the release into the circulation of gastrointestinal peptides associated with satiety (Wren et al. 2007). The weight reduction after treatment is related to the dual action of mitratapide, namely decreased uptake of dietary lipids and decreased appetite.

Glucose tolerance tests at baseline showed for five obese dogs impaired insulin sensitivity compared to historical glucose tolerance test results in the same dogs with ideal body weight. This is an expected effect of obesity (Goschke 1977). After the mitratapide treatment schedule, insulin sensitivity was increased again in four out of the five dogs. These data suggest that obese dogs with insulin resistance may benefit from weight loss (Leray et al. 2008).

Fig. 6 Mean feed consumption \pm SD before, during and at the end of the mitratapide treatment

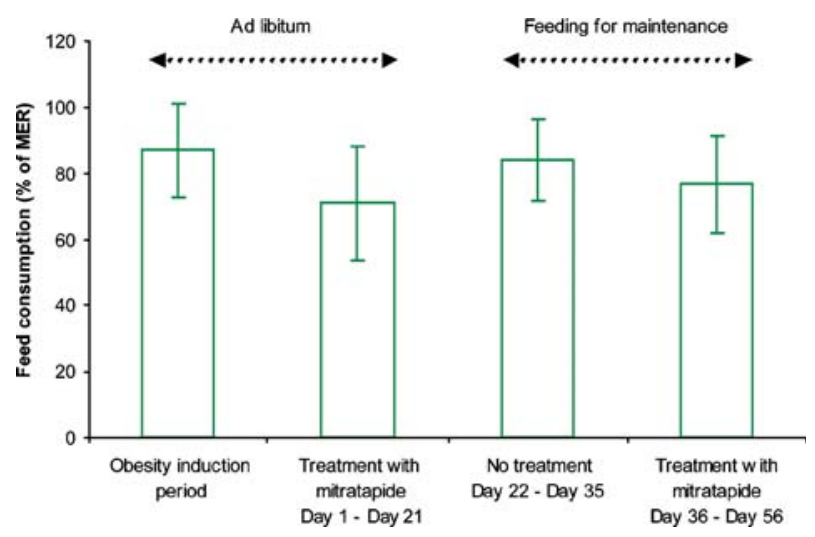


As indicated above, fat tissue is very metabolically active and produces a wide range of hormones (adipokines, leptin, resistin) (Greco 2008). Leptin plays a role in the pathogenesis of articular degenerative diseases such as osteoarthritis (Lago et al. 2008). In humans was recently demonstrated that obesity has a significant impact on the musculoskeletal system being associated with both degenerative and inflammatory conditions (Anandacoomarasamy et al. 2009). Thus, mechanical stress together with biochemical factors is thought to contribute to the onset of osteoarthritis. Mitratapide treatment was demonstrated to reduce significant weight loss and normalizes the percentage body fat. This may be beneficial not only in obese dogs with osteoarthritis but may also prevent the onset of osteoarthritis in overweight and obese dogs and is therefore considered to produce a significant health benefit.

Reduced faeces quality and vomiting is considered non-related to the treatment because it also occurred during non-treatment periods. The case of pseudo-pregnancy is unlikely to be related to mitratapide treatment. Neither negative energy balance nor reduced fat absorption is associated with pseudopregnancy. In addition, in our colony pseudopregnancies occur occasionally in bitches independent of treatment with mitratapide. The latter is also true for otitis externa. However, excess adipose tissue is known to be associated with reduced immunity (Dixit 2008) what might be a factor in this case.

This study demonstrated that the application of mitratapide treatment is safe for the animal. During the study no serious adverse reactions were seen.

\section{Conclusions}

From this study it can be concluded that weight loss induced by the treatment of obese dogs with mitratapide is the result of a loss of adipose tissue. After a treatment schedule with mitratapide, the body fat percentage of obese dogs had returned within the normal range. The amount of lean tissue and mineral bone content is hardly affected. As obesity is one of the most common underlying causes of osteoarthritis and several hormones produced by adipose tissue are known to play a major role in the occurrence of comorbidities of obesity, normalizing the percentage of body fat will produce a significant health benefit.

This study also suggested that in obese dogs with marked insulin resistance, losing weight with mitratapide might help to reverse the insulin resistance.

Open Access This article is distributed under the terms of the Creative Commons Attribution Noncommercial License which permits any noncommercial use, distribution, and reproduction in any medium, provided the original author(s) and source are credited.

\section{References}

Anandacoomarasamy A, Fransen M, March L (2009) Obesity and the musculoskeletal system. Current Opinion in Rheumatology 21(1):71-77.

Armstrong PJ, Churchill JA (2008) Fat - the largest endocrine organ: not just energy storage after all! (abstract). 80 th Western Veterinary Conference, Las Vegas, NV, USA. 
Belfiore F, Iannello S, Camuto M, Fagone S, Cavaleri A (2001) Insulin sensitivity of blood glucose versus insulin sensitivity of blood free fatty acids in normal, obese, and obese-diabetic subjects. Metabolism 50 (5):573-582.

Burkholder W, Bauer J (1998) Foods and techniques for managing obesity in companion animals. Journal of the American Veterinary Medical Association 212: 658-662.

Burkholder W, Toll P (1997) Obesity. In: Hand, Tacher, Remillard, Roudebush (Ed.) Small Animal Clinical Nutrition, pp 401-430.

Dixit VD (2008) Adipose-immune interactions during obesity and caloric restriction: reciprocal mechanisms regulating immunity and health span. Journal of Leukocyte Biology 84(4):882-892.

Edney A, Smith P (1986) Study of obesity in dogs visiting veterinary practices in the United Kingdom. Veterinary Record 118:391-396.

Goldstein DJ (1992) Beneficial health effects of modest weight loss. International Journal of Obesity \& Related Metabolic Disorders: Journal of the International Association for the Study of Obesity 16:397415.

Goschke H (1977) Mechanism of glucose intolerance during fasting: differences between lean and obese subjects. Metabolism: Clinical \& Experimental 26:1147-1153.

Greco DS (2008) Fat as an endocrine organ (abstract). 80 th Western Veterinary Conference, Las Vegas, NV, USA.

Kealy RD, Lawler DF, Ballam JM, Mantz SL, Biery DN, Greeley EH, Lust G, Segre M, Smith GK, Stowe HD (2002) Effects of diet restriction on life span and age-related changes in dogs. Journal of the American Veterinary Medical Association 220:1315-1320.

Laflamme DP (1997) Development and validation of a body condition score system for dogs. Canine Practice 22(4):10-15.

Lago R, Gómez R, Lago F, Gómez-Reino J, Gualillo O (2008) Leptin beyond body weight regulation-current concepts concerning its role in immune function and inflammation. Cellular Immunology 252(1-2):139145.

Lawler DF, Evans RH, Larson BT, Spitznagel EL, Ellersieck MR, Kealy RD (2005) Influence of lifetime food restriction on causes, time, and predictors of death in dogs. Journal of the American Veterinary Medical Association 226:225-231.

Leray V, Serisier S, Khosniat S, Martin L, Dumon H, Nguyen P (2008) Adipose tissue gene expression in obese dogs after weight loss. Journal of Animal Physiology and Animal Nutrition.92(3):390-398.

McGreevy PD, Thomson PC, Pride C, Fawcett A, Grassi T, Jones B (2005) Prevalence of obesity in dogs examined by Australian veterinary practices and the risk factors involved. Veterinary Record 156 (22):695-702.

Mertens IL, Van Gaal LF (2000) Overweight, obesity, and blood pressure: the effects of modest weight reduction. Obesity Research 8:270-278.

NRC (2006) Nutrient requirements of dogs and cats. The national Research Council. National Academic Press, Washington D.C., USA.

Pi-Sunyer FX (1996) A review of long-term studies evaluating the efficacy of weight loss in ameliorating disorders associated with obesity. Clinical Therapeutics 18: 1006-1035.

Speakman JR, Booles D, Butterwick R (2001) Validation of dual energy X-ray absorptiometry (DXA) by comparison with chemical analysis of dogs and cats. International Journal of Obesity \& Related Metabolic Disorders 25:439-447.

Wing RR, Koeske R, Epstein LH, Nowalk MP, Gooding W, Becker D (1987) Long-term effects of modest weight loss in type II diabetic patients. Archives of Internal Medicine 147:1749-1753.

Wren JA, Gossellin J, Sunderland SJ (2007) Dirlotapide: a review of its properties and role in the management of obesity on dogs. Journal of Veterinary Pharmacology and Therapeutics Suppl 1:11-16. 